http://jmscr.igmpublication.org/home/

ISSN (e)-2347-176x ISSN (p) 2455-0450

crossref DOI: https://dx.doi.org/10.18535/jmscr/v7i11.67

Journal Of Medical Science And Clinical Research

\title{
Evaluation of female infertility with endometrial PCR for tuberculosis in a district tertiary centre
}

\author{
Authors \\ Dr S.Bhanu Rekha M.D ${ }^{1}$, Dr Kushanapalli Anusha M.S. ${ }^{2}$ \\ ${ }^{1}$ Associate Professor, Dept of Obstetrics \& Gynaecology, SVS Medical College \& Hospital, Mahabubnagar, \\ Telangana, India 509001 \\ ${ }^{2}$ Obstetrician \& Gynaecologist, Fertility Consultant, Nandhini Hospital, Warangal, Telangana, India
}

\begin{abstract}
Introduction: Female Genital tract tuberculosis is one of the most common causes of infertility and often asymptomatic before it ends up with gross damage to the fallopian tubes and endometrium and ovaries. Hence a routine screening of infertile women with a reasonable good method of diagnosis like Polymerase Chain Reaction (PCR) is mandatory in the infertility evaluation in females Objective - to evaluate infertile women for tuberculosis using TB PCR.

Results: Of the 100 women who came for infertility evaluation during a period of 2 years in a infertility clinic, 16 of them turned out to be positive for tuberculosis by TB PCR.

Conclusion: Endometrial PCR still remains the most effective and rapid diagnostic test for genital tuberculosis in the evaluation of female infertility.

Keywords: Tuberculosis, Polymerase Chain Reaction, Endometrium.
\end{abstract}

\section{Introduction}

Infertility defined as inability to conceive spontaneously even after one year of unprotected intercourse. Female Genital tuberculosis (FGTB) prevalence ranges from 15 to $18 \%$ in $\operatorname{India}^{(1)}$. FGTB results in multiple causes of infertility, major cause being Fallopian tubal obstruction and dysfunction, the uterine endometrium TB leading to implantation failure due to thinning and adhesions and also ovarian involvement rarely adds up to the causes of infertility in females. The most common group of females affected are in late adolescence to 40 years. The FGTB is usually secondary to the silent bacillemia primarily from other organs like Lungs, intestines, abdominal TB. The common sites of FGTB are the Fallopian tubes (90-100\%), endometrium $(50 \%)$ ovaries (15 $-30 \%)$, cervix (5\%), vulva and Vagina (1\%). The patients are usually asymptomatic and hence the diagnosis of FGTB is usually made during the evaluation of infertility or during the evaluation of menstrual irregularities or rarely as an non healing ulcer on vulva or vagina usually by clinical suspicion. The diagnostic modalities like Mantoux and ESR are usually nonspecific. The AFB culture takes a long time over 4 weeks and low detection rate sometimes. BACTEC allows rapid detection. Sometimes the diagnosis of FGTB is made indirectly with the help of HSG though HSG is not done in highly suspicious condition of FGTB for the fear of exacerbation ${ }^{(2)}$. USG or CT 
abdomen and Pelvis is usually useful only in cases of organised pelvic mass.

Hysterolaparosopy is recommended for the confirmation of FGTB as it can detect tubercles, peritubal adhesions, hydrosalpinx and Tubo ovarian masses and any Uterine synaechea. This is also useful for endometrial sampling and for taking out suspicious areas for Histopathology (HPE) and for Polymerase Chain Reaction.

HPE alone can sometimes have s high false negativity due to inability to collect the sample from right place and time.

Polymerase Chain Reaction (PCR) is a reasonably sensitive technique available where in it can detect fewer than ten organisms in clinical specimen which is usually seen in paucibacillary condition like FGTB. This study was attempted to diagnose FGTB by PCR through endometrial sampling done either as an office procedure by endometrial biopsy or done as a routine during Hysterolaparoscopy.

\section{Aims and Objectives}

To evaluate the infertile women for genital tract tuberculosis by subjecting Endometrial biopsy for Polymerase Chain Reaction

\section{Material \& Methods}

Around 100 women who presented for infertility evaluation over a period of two years between august 2017 to July 2019 in a tertiary teaching hospital in mahabubnagar, were offered endometrial PCR either as a part of next line of investigation after Mantoux positive status done as a routine infertility workup for endometrial tuberculosis by endometrial pipelle biopsy or the sample obtained by endometrial scraping during hysterolaparoscopy procedure.

\section{Results}

The present study was carried out in the Dept of Obstetrics and Gynaecology at a tertiary teaching hospital, mahabubnagar district, Telangana from August 2017 to July 2019 and total of 100 women who underwent endometrial biopsy either as a work up for mantoux positive state or during diagnostic Hysterolaparoscopy procedure, both done as a part of infertility work up.

The diagnosis of genital tuberculosis was confirmed in 16 of the 100 enrolled cases (16\%) by TB PCR. among these 16 cases, 6 patients were from hysterolaparoscopy group and 10 were from the patients who underwent endometrial pipelling in the premenstrual period. The clinical and hysterolaparoscopy findings were analysed and co related with the positive TB PCR. Among the patients who underwent Hysterolaparoscopy, there was delayed spillage of dye, small multiple tubercles, and peritubal and pelvic adhesions, extravasation of dye and TO mass in 4 to $5 \%$ of patients (2). Positive cases in TB PCR were also correlated with the routine HPE of the endometrial sample sent simultaneously in 6 patients. HPE was positive only in 2 cases $(12.5 \%)$. Of the 16 women who were positive for TB PCR, 12 of them were primary infertility and 4 (4\%) were secondary infertility. Majority of these positive women, $14(87.5 \%)$ in the age group of $21-30 \mathrm{yrs}$ and $(12.5 \%)$ were in the age group of $31-40$ yrs. Of the 16 women, majority were asymptomatic $(75 \%)$ and only 4 were complaining of scanty menses. There was a history of Tuberculosis of lungs as a primary diagnosed in 1woman (12.5\%) before her marriage and the patient had discontinued the treatment after 3 months of ATT.

\section{Discussion}

Genital Tuberculosis is one of the deadly silent diseases affecting women desiring conception all over the world. In India the incidence of FGTB has doubled $19 \%$ in 2011 to $30 \%$ in $2015^{(3)}$. In the present study, the incidence of Genital TB in infertile women in our study period was $16 \%$. Most of the women who enrolled for infertility evaluation were asymptomatic and were routinely investigated with Mantoux, where in only a positive Mantoux patient was further asked to follow up with endometrial biopsy for TBPCR and HPE. Few of the patients who were undergoing Diagnostic Hysterolaparoscopy as a 
infertility evaluation were also enrolled for this study by investigating endometrial curetting for TBPCR and /or HPE. Positive PCR for Tuberculosis and granulomatous lesions in HPE were taken as positive in diagnosing Genital Tuberculosis. In our study, almost $94 \%$ cases were diagnosed using TB PCR due to the high pickup rate of nested PCR.

6 patients who underwent hysterolaparoscopy had normal spillage except for $30 \%$ of them who had delayed spillage as fallopian tubes are said to be affected primarily affected in FGTB. These patients presented with delayed or absent spillage of dye in almost $45 \%$ cases just like in other studies $^{(2)}$. around $10 \%$ patients also presented with peritubal and omental adhesions. Hysteroscopy also demonstrated intrauterine synechiae in $10 \%$ patients.

$75 \%$ women in this study group had normal menstrual cycle whereas $25 \%$ had scanty menstruation. This incidence of hypomenorrhea is quite like the Indian study by ${ }^{(5)}$

\section{Conclusion}

Genital tuberculosis is the most important under evaluated causes in a developing country like India leading to infertility in these young women and also menstrual irregularity and chronic abdominal pain and hence affecting the quality of life in women. Hence arises a necessity to diagnose with a quick and reliable method.

Since FGTB is the paucibacillary type, TBPCR is still the most preferred diagnostic tool as it is easy, rapid, highly sensitive method of diagnosing Genital TB.

\section{References}

1. Varma TR. Genital tuberculosis and subsequent fertility. Int J GynaecolObstet 1991;35:1-11

2. Sharma JB, Roy KK, Pushparaj M. Kumar S, Malhotra N, Mittal S. Laparoscopic findings in female genital tuberculosis. Arch Gynecol Obstet.2008;278(4):359-64.

3. Sharma J, Gupta Nupur, Roy Kallol, Mittal Suneeta, Jain Sunesh, Neyaz Zafaretal. Hysterosaphingographic findings in infertile women with genital tuberculosis. Int J of Gynecol and Obstet. 2008;150-155.

4. Gupta N, Sharma J.B., Mittal S, Misra R, Kukreja M. Genital tuberculosis in Indian infertility patients. Int $\mathbf{J}$ of Gynaecol and Obstet. 2007;97(2):135-138.

5. Oosthuizen, A.P., Wessels P.H., Heferh J.N. Tuberculosis of female genital Tractin patients attending an infertility clinic. South African Medical Journal.1990; 77:562-564. 